\title{
Performance diagnostics in endurance sports - anaeroboc threshold, VO2max, aerobic capacity \\ - where do we go?
}

\section{ELITE SPORTS / EXERCISE PHYSIOLOGY / PERFORMANCE / SPORTS SCIENCE}

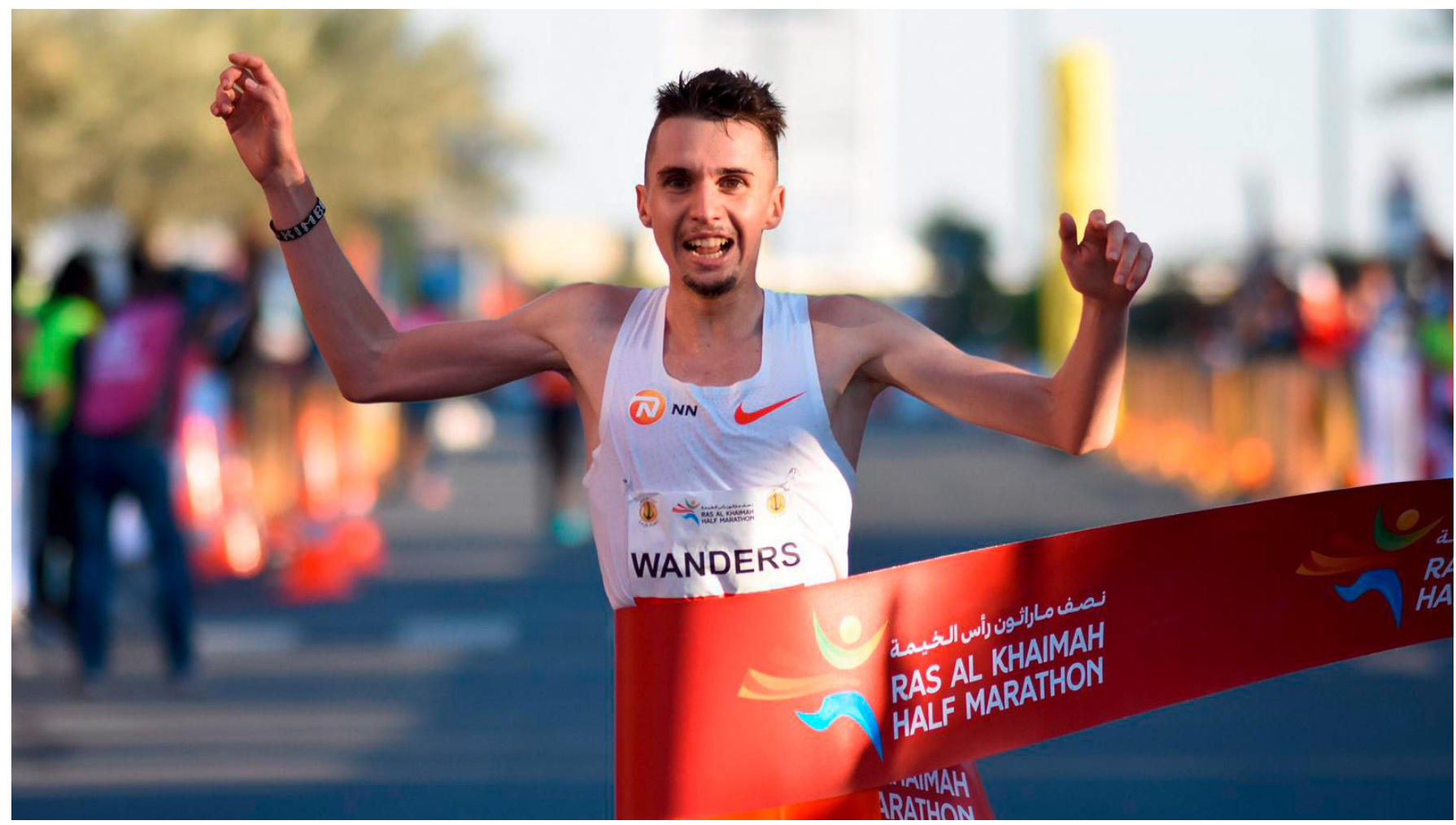

Leistungsdiagnostik im Ausdauersport - anaerobe Schwelle, VO $\square$ max, aerobe Kapazität - wohin geht die Reise?

\section{Clénin G}

Sportmedizinisches Zentrum Bern-Ittigen, Haus des Sports, Switzerland

\section{Abstract}

Exercise testing in athletes and patients is an important and valuable diagnostic tool in the hand of the physician and sport scientist. A close collaboration between sport scientist and sport physician creates a win-win-win-situation for athletes and patients and professionals equally. According to the demands of the sport an incremental lactate threshold test (determination of the anaerobic threshold), testing of $\mathrm{VO}_{2} \max$ 
and performance at $\mathrm{VO}_{2} \max$, and a testing of aerobic capacity (performance at competitional level) are the tests of choice. All tests need to be valid, reliable and sport specific. Sport specificity in testing is for practical purposes the most important.

\section{Zusammenfassung}

Leistungsdiagnostische Tests bei Athleten und Patienten sind in der Hand des Sportmediziners/Sportwissenschafters ein wichtiges und wertvolles Tool zur Diagnostik und Trainingsberatung. Eine enge Zusammenarbeit zwischen Sportwissenschafter und Sportmediziner schafft für Athleten und Patienten wie die Fachpersonen eine Win-win-win-Situation. Je nach Anforderungsprofil der Sportart sind ein Laktatstufentest (Bestimmung der anaeroben Schwelle), eine Testung der $\mathrm{VO}_{2} \max$ und der Leistung an der $\mathrm{VO}_{2}$ max sowie ein Kapazitätstest (Tempohärte mit Wettkampfbelastung) die Tests der Wahl. Alle Testungen sollen sportartspezifisch, valide und reliabel sein. Die Sportartspezifität hat für die praktische Aussagekraft der Testung am meisten Gewicht.

\section{Julien Wanders, erfolgreicher Schweizer Langstreckenläufer. Welche Rekorde wird er in Zukunft aufstellen können?}

(Quelle: Bild freundlicherweise vom Athleten zur Verfügung gestellt)

\section{Einleitung/Ziele der Leistungsdiagnostik}

Ausdauersport hat zahlreiche bekannte positive Auswirkungen auf den menschlichen Organismus [1,2,3]. Ausdauersportarten sind aber auch Wettkampfdisziplinen in zahlreichen olympischen und nichtolympischen Sportarten. Sowohl leistungssportliche Athleten wie auch ambitionierte Freizeitsportler absolvieren bereits in der Kinder- und Jugendzeit und im Erwachsenenalter ein regelmässiges Ausdauertraining mit dem Ziel, ihre Ausdauerleistungsfähigkeit zu verbessern. Die Leistungsdiagnostik kann sie in diesem Setting in wertvoller Art und Weise unterstützen, indem in den folgenden vier Bereichen Rückmeldungen und Empfehlungen abgegeben werden können (adaptiert aus Leistungsdiagnostik Manual Swiss Olympic 2015; 4):

1. Erhebung des aktuellen Leistungsstandes.

Dient der Erstellung eines Stärke-Schwäche-Profils und dem Vergleich mit anderen Athletinnen (z.B. innerhalb eines Kaders, nationaler Vergleich oder Vergleich mit Weltspitze).

2. Beurteilung der Leistungsentwicklung.

Ermöglicht das detaillierte Erkennen des intraindividuellen Leistungsfortschrittes. Dies ist insbesondere bei langwierigen Verletzungen oder bei Operationen mit nachfolgender Rehabilitation und der Begleitung im Return-

to-Sports wertvoll.

3. Optimierung der Trainingssteuerung. 
Die ermittelten Messparameter können sportartspezifisch zur Trainingssteuerung eingesetzt werden, wie z.B. die anaerobe Schwelle zur Bestimmung der Trainingszonen.

4. Abschätzung des Leistungspotenzials.

Mit dem Ziel, Schlüssel-Parameter zu messen, welche zu einem grossen Teil genetisch determiniert sind (z.B. Hämoglobinmasse und $\mathrm{VO}_{2} \max$ im Ausdauersport).

\section{Umgang mit Hitze, Reisen, Infekte und Höhe}

In den vergangenen zwanzig Jahren wurde im Ausdauersport viel dazugelernt, unter anderem: Wie bereitet man sich in extremer Hitze mit Akklimatisation und Vorkühlung optimal auf einen Wettkampf in diesen Temperaturen vor [5-9]. Oder wie man auf wiederholten langen Flugreisen mit Aufenthalt in verschiedenen Zeit- und Klimazonen gesund bleibt, dem Jetlag möglichst ausweicht und die geeigneten Massnahmen zur Infektprävention anwendet [5,9,10,11].

Auch Höhentraining erlebte vor allem in den Ausdauer- und einigen Teamsportarten ein Revival und gewann wieder an Popularität. In den allermeisten Fällen mit dem Living-High-Training-Low-Prinzip mehr (oder auch mal weniger) erfolgreich praktiziert [12-16]. Man erlernte die Feinheiten des idealen langsamen Trainingsstarts in den ersten Tagen auf der Höhe, der Wichtigkeit des Gesundbleibens und des idealen Timings der Performance, z.B. ab dem 10. Tag nach dem Höhentrainingsaufenthalt, um nur einige der Merkpunkte des erfolgreichen Höhentrainingsaufenthaltes zu erwähnen [5,9,12].

\section{Neue Strömungen und Trends im Trainingsbereich}

Auch im Training selber gab es diverse Strömungen und Trainingstheorien, welche diskutiert und auch praktiziert wurden: Der klassische Approach mit sehr viel Grundlagen-, einigen Schwellentempo- und vereinzelten Intervallbelastungen. Hin zum polarisierten Training, welches die Belastungen im Mitteltempobereich bewusst auslassen will, «um nicht alles mittelmässig zu trainieren, die lockeren Einheiten zu schnell und die eigentlich überschwelligen Belastungen zu langsam» [5,12-17]. Im polarisierten Training wird der Focus daraufgelegt, dass die grosse Mehrheit der Trainings (z.B. 80-85\% der Trainingszeit) im locker-extensiven Bereich stattfindet mit einem kleineren Anteil (15-20\%) hochqualitativer Einheiten im Bereich der anaeroben Schwelle und Wettkampftempo und bewusst auch überschwelligen Einheiten [17,18]. Vom HIIT, High Intensity Intervall Training, wird berichtet, dass es bereits um 1970 praktiziert wurde [21]. Es gewann mit den Arbeiten von Tabata 1996 mehr Bedeutung und Aufmerksamkeit [22]. Die Trainingsform mit einer oder mehreren Serien von kurzen hochintensiven, klar überschwelligen Belastungen mit kurzen Pausen dazwischen, wurde auch als selbständige Trainingsmodalität interessant, weil es damit gelang, die maximale Leistungsfähigkeit und auch die $\mathrm{VO}_{2}$ max zu verbessern [5,22,23]. Dass für einen Leistungssportler diese Trainingsform so nicht alleine stehen kann, liegt auf der Hand. Seiler kommt in seiner Zusammenfassung zu folgenden Schlüssen: «Athletes respond individually to training. An 80-20 ratio of Low to Threshold/High intensity training is common and apparently gives excellent long-term results among endurance athletes. High intensity training is a critical component of the training of all succesful endurance athletes. About two high intensity training sessions per week seem to strike the good balance between positive effects and stress load.» (in 5; 
S. Seiler Training intensity distribution, p. 38).

Neben der Rumpfkraft und segmentalen Stabilisation, welche in der Schweiz richtigerweise für jeden leistungssportlich Aktiven gefordert und gefördert wird, wurde sogar im Rad- und Laufsport nach und nach das sportartspezifische Krafttraining salonfähig. Sportartspezifisches Krafttraining wird besonders in der Off-Season, mittlerweile aber auch ganzjährig, in die Trainingspläne von den allermeisten SpitzenAusdauersportlern integriert [5,24,25].

\section{Wandel der Disziplinen im Ausdauersport}

Auch der Ausdauersport selber wandelte sich: Die langen Ausdauerformate gibt es noch mit Marathon im Laufsport, 50 und $30 \mathrm{~km}$ im Langlauf, Radrundfahrten und Classiquen, olympische Distanz und MarathonDistanz Mountainbike, Orientierungslauf-Langdistanz und Triathlon von der olympischen Distanz bis zum Ironman. Aber viele Sportverbände entwickelten neue zuschauerfreundliche und medienwirksamere Formate mit Sprint-Entscheidungen, welche zentral und puplikumsnah zu besten TV-Sendezeiten den Sport in die Big Cities und in die Fernsehstuben trugen. Dieser Trend wird sich publikumsseitig weiter verstärken, indem die Beiträge mit den sportlichen Entscheidungen in noch kürzeren Spots prägnant fürs Internet aufbereitet werden müssen, damit sie mit PC und Smartphone verfolgt werden können. So kennen Langlauf, Mountainbike, Triathlon, Orientierungslauf, Biathlon u.a.m. bereits diese Sprintformate und sind daran, diese eher noch auszubauen. Dies bringt eine weitere Diversifizierung der Sportarten mit neuen Anforderungsprofilen. Es gilt nun mehrere, oftmals wenige Minuten dauernden Vorläufe, Viertelund Halbfinalruns zu überstehen, bevor im Final um den Tagessieg mitgekämpft werden kann. Die organisierenden Sportverbände beobachten ihre eigenen Formate und die damit verbundene Entwicklung des Sports. Und entsprechend reagieren die nationalen Teams, indem sie Sprint-Trainingsgruppen bilden und sich spezialisieren. Gleiches gilt für die Athleten, welche sich je nach Wettkampfplanung und eigenen Fähigkeiten noch spezifischer für diese Wettkämpfe vorbereiten oder sie auch bewusst auslassen, um Kraft und Energie zu sparen.

\section{Welche Leistungstests für welchen Zweck?}

Die Physiologie des Menschen ist selbstverständlich immer noch die Gleiche wie vor zwanzig Jahren, aber die Anforderungen des Sports haben sich teilweise geändert, sich diversifiziert. So ist es naheliegend, dass die Leistungsdiagnostik dieses breitere Anforderungsprofil abzubilden versucht. Hierzu reicht ein einzelner Test in der Regel nicht aus, will man Aussagen über verschiedene Kenngrössen im Ausdauersport treffen. Typischerweise werden je nach Fragestellung zwei oder drei der nachfolgenden Tests ausgewählt [4,5]:

- Laktatstufentest zur Bestimmung der anaeroben Schwelle und der Trainingszonen

- $\mathrm{VO}_{2}$ max-Test zur Bestimmung der maximalen Sauerstoffaufnahme und der maximalen Leistungsfähigkeit

- Kapazitätstest zur Bestimmung der Tempofestigkeit und Überprüfung der Wettkampfgeschwindigkeit

Alle drei Tests müssen so sportartspezifisch wie nur möglich und standardisiert durchgeführt werden 
[4,5,25]. In verschiedenen Ausdauersportarten hat sich durchgesetzt, dass die Testung bewusst breiter erfolgt, d.h. diese grundsätzlich als Maximaltests konzipierten Testprotokolle werden in einer bestimmten sinnvollen Abfolge an Testdays absolviert. So finden z.B. am Testtag 1 ein nicht ganz maximal durchgeführter Laktatstufentest und anschliessend die $\mathrm{VO}_{2}$ max-Testung statt. Am Testtag 2 folgt dann der wettkampfspezifische Kapazitätstest.

\section{Anaerobe Schwelle im Laktatstufentest}

In den Ausdauersportarten ist der Laktatstufentest im standardisierten Protokoll mit Bestimmung der maximalen Leistung beim Abbruch und der Ermittlung der anaeroben Schwelle nach wie vor der klassische Basistest [4,5,25]. Die anaerobe Schwelle entspricht der höchsten Belastung (in km/h Geschwindigkeit oder in Watt Leistung), bei welcher noch ein Gleichgewicht zwischen Laktatproduktion und Laktatelimination, also ein Laktat-Steady-State gewährleistet ist [4,25]. Da für die echte Bestimmung des MaxLaSS, also des Maximal Lactate Steady State, mehrere Tests über mehrere Tage notwendig wären, hat sich der Laktatstufentest letztlich durchgesetzt. Der Laktatstufentest liefert mit den Kenngrössen der anaeroben Schwellenleistung und der Abbruchleistung gleichermassen reliable wie valide Daten zur Beurteilung der Ausdauerleistungsfähigkeit [26,27,28]. Hierbei scheint die Abbruchleistung der Schwellenleistung gemäss Literatur mindestens ebenbürtig. Ebenso liefert der Laktatstufentest geeignete Messgrössen für einen intra- und interindividuellen Quer- und Längsvergleich und ist aussagekräftig zur Bestimmung der Trainingszonen [4,25,28].

Auch im Radsport wird der Laktatstufentest als Basistest verwendet. Mit der Entwicklung der mobilen Powermeter auf dem Rennrad hat aber auch das Testverfahren «Critical-Power-Konzept» eine grössere Verbreitung gefunden [29,30,31, 32]. Es geht hierbei um die Ermittlung der maximal möglichen Durchschnittsleistung für eine gegebene Zeit. Am meisten interessiert die Critical Power über 60 min (CP60), also die maximal mögliche Durchschnittsleistung über $1 \mathrm{~h}$. Die Testung besteht allerdings oftmals in einer Annäherung dieser Grösse, indem ein oder manchmal zwei All-Out-Tests über 5 min und 20 min die CP5 und CP20 gemessen und dann die CP60 rechnerisch ermittelt wird. Letztlich aus dem Grund, da die Bestimmung der CP60 aufwändig, belastend und stark motivationsabhängig ist. Als Erfahrungswert kann gelten, dass die CP60 in etwa der Leistung an der anaeroben Schwelle entspricht [4]. Aufgrund der CP60 lassen sich Trainingsempfehlungen für ein Watt-gesteuertes Training mit dem mobilen Powermeter ableiten. Die Testung kann mit dem eigenen Rennrad und dem eigenen Powermeter in der gewohnten Umgebung als Feldtest ohne grossen Aufwand durchgeführt werden. Das Wegfallen von Transferfehlern von der Testung ins Training und die einfache und gute Bestimmung der Trainingszonen ohne Laktatmessung sind Vorteile des Critical-Power-Konzepts [4,30,31,32]. Als wichtige Überlegung bei der Festlegung der Standardisierung von Protokollen geht es darum zu überlegen, was die ideale Stufendauer und das optimale Belastungsinkrement eines Laktatstufentest sind. Grundsätzlich kann festgehalten werden, dass wie im Leistungsdiagnostik Manual Swiss Olympic vorgeschlagen, die ideale Stufendauer zwischen 3-5 min liegt und das Inkrement für Geschwindigkeit von 1-2 km/h bei einem Lauftest und das Leistungsinkrement 20-50 Watt bei einem Radtest betragen sollte [4]. In vielen sportmedizinischen Zentren der Schweiz werden nach wie vor die Stufendauer von 3 min und die Belastungsinkremente von 1,8 km/h fürs Laufen und 30 Watt fürs Radfahren verwendet. Zur Illustration der typischen Auswirkungen der Veränderung der Stufendauer und/oder des 
Belastungsinkrementes auf Laktat und Herzfrequenz hilft uns die Grafik 1 aus dem Leistungsdiagnostik Manual Swiss Olympic.

Eine wichtige Erkenntnis, welche sich in der Umsetzung ebenfalls lohnt, ist die konsequente Anwendung einer ganz geringen Laufbandsteigung für den Laktatstufentest im Laufen, um den im Labor fehlenden Luftwiderstand zu kompensieren. Mit 1\% Steigungswinkel des Laufbandes wird im laufsportrelevanten Geschwindigkeitsbereich diese sonst entstehende Differenz mit im Labor zu hohen Geschwindigkeiten gut ausgeglichen $[4,33)$.

\section{Individuell anaerobe Schwelle IANS vs. D-max modifiziert oder gar beide?}

Zur Bestimmung der anaeroben Schwelle wurden zahlreiche Modelle vorgeschlagen. Ausgehend von der alten $4 \mathrm{mmol} / \mathrm{L}-\mathrm{Methode}$ nach Mader wurden multiple andere Berechnungsverfahren und auch andere Messprotokolle wie zum Beispiel der Laktatsenke-Test propagiert [34,4]. Letztlich schien keines dieser mathematischen Modelle oder Messverfahren wirklich überlegen, so dass sich in der Schweiz richtigerweise die pragmatische und athletenzentrierte Methode mit der Bestimmung der sogenannten individuellen anaeroben Schwelle (IANS) durchsetzte [35]. Sie fand dann auch Eingang in das Manual Ausdauerleistungsdiagnostik von Swiss Olympic 2003 und war bis vor Kurzem der Standard in der Schwellenbestimmung. Im Gegensatz zu den vorgenannten messtechnisch und auch mathematisch etwas anfälligen Modellen bietet die IANS dem erfahrenen Testleiter den Vorteil, den Athleten gemäss dem untenstehenden Algorhythmus in seinem Leistungsvermögen individuell gut einzuschätzen [35,4].
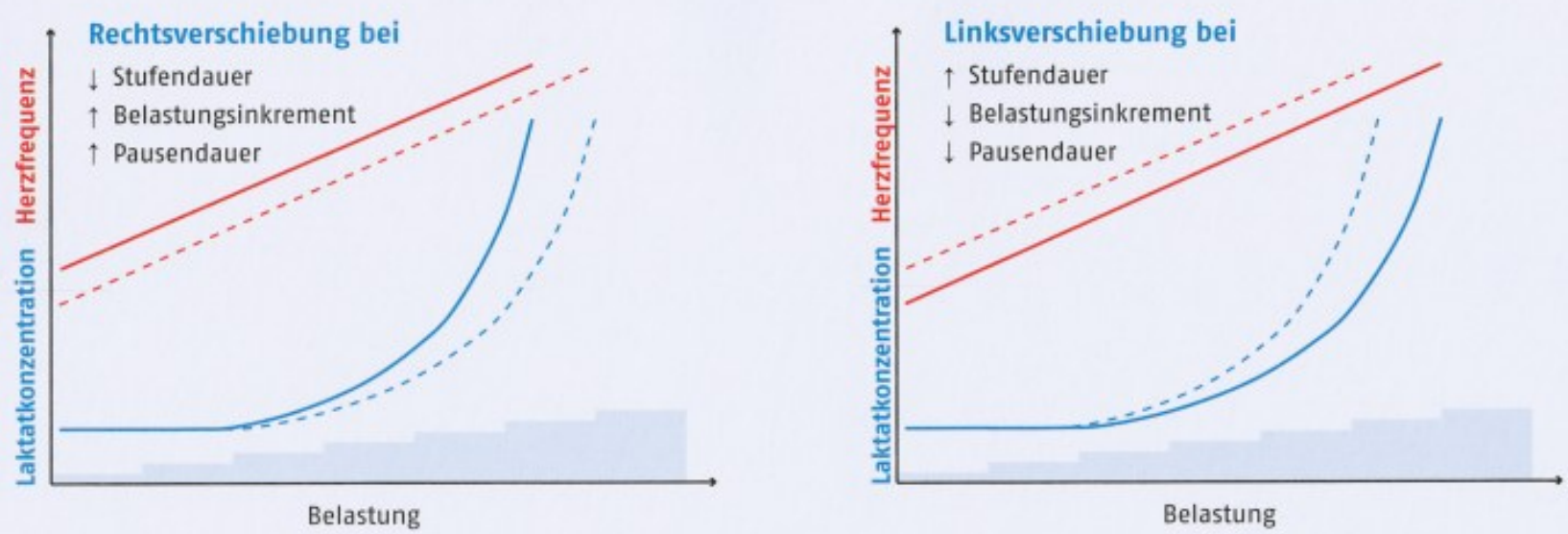

Ausgangslage Jeweiliger Fall

Grafik 1: Einfluss von Veränderung der Stufendauer, des Belastungsinkrementes und der Pausendauer auf die Laktatund Herzfrequenz-Leistungskurve (Quelle: Leistungsdiagnostik Manual Swiss Olympic, 4, S. 26). 


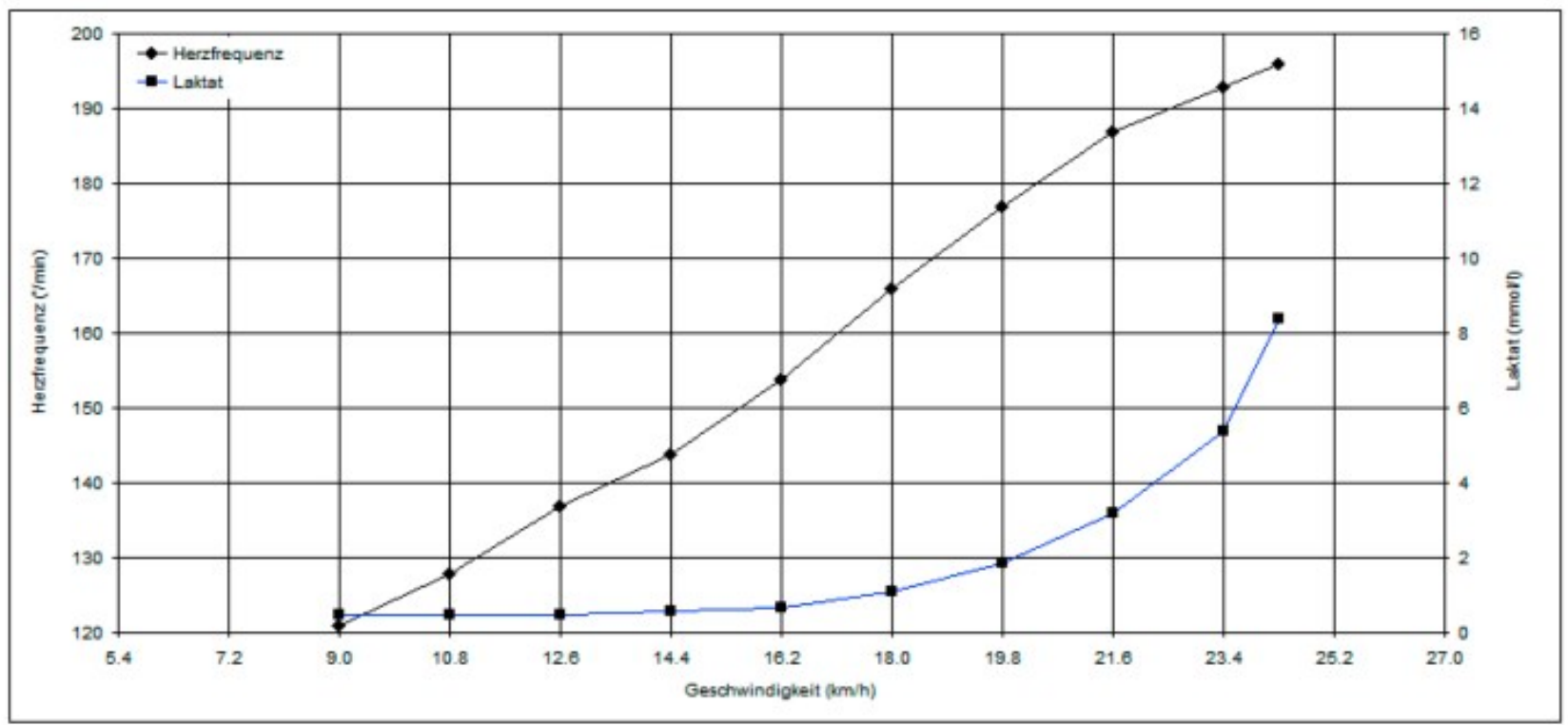

\section{Zusammenfassung}
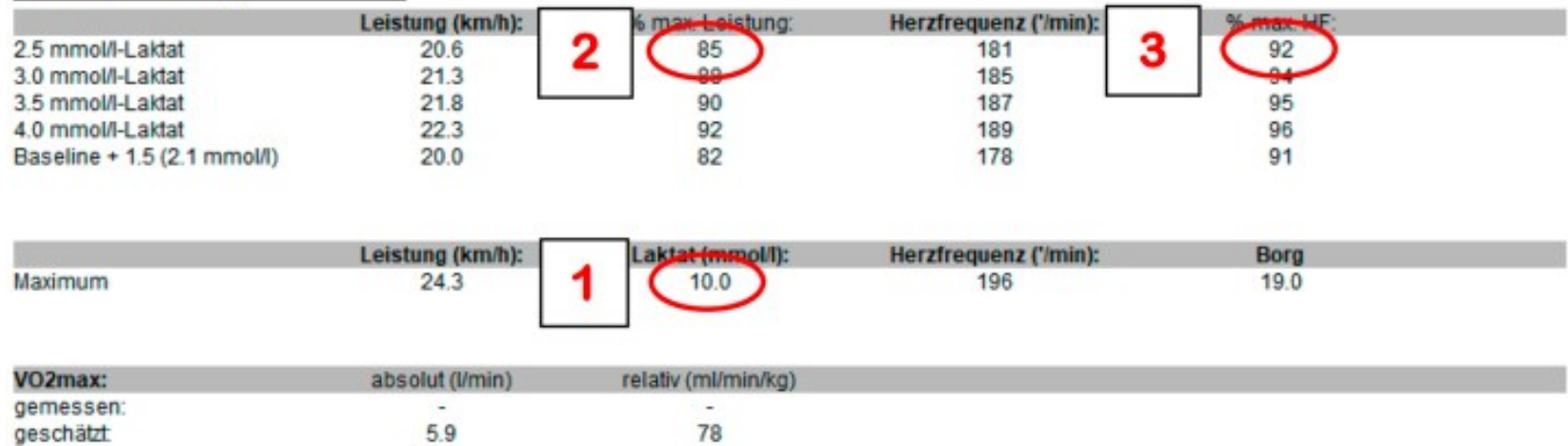

$\begin{array}{lll}\text { geschätzt } & 5.9 & 78\end{array}$

\section{Schwellenbestimmung}

Schwellentyp:

Grafik 2: Bestimmung der IANS mit den Kriterien 1. Laktat: bei 10mmol/1 Korrektur möglich aber nicht zwingend erforderlich, die anderen drei Kriterien drängen aber eine Korrektur auf. 2. Herzfrequenz 90-95\%. 3. \% der maximalen Leistung 82-86\%. 4. Borg 14-16.

\section{Bestimmung der individuell anaeroben Schwelle IANS (35)}

Zur Ermittlung der anaeroben Schwelle werden folgende Punkte berücksichtigt:

\section{Laktat:}

Bei Maxlaktatwerten $<8 \mathrm{mmol} / \mathrm{l}$ soll der Schwellenlaktatwert ausgehend von $4 \mathrm{mmol} / \mathrm{l}$ gegen unten*, bei Maxlaktat $>12 \mathrm{mmol} / \mathrm{l}$ gegen oben korrigiert werden. *Voraussetzung gute Ausbelastung durch 1 . Eindruck des Testleiters, 2. Borg maximal $\geq 18$.

2. Herzfrequenz: 
Üblicherweise 10-20 Schläge (90-95\% max Hf) unter der maximalen Herzfrequenz. Sehr gut Ausdauertrainierte haben ihre Hf an der IANS näher bei der Max Hf, wenig Trainierte weiter davon entfernt.

3. Prozent der max. Leistung an der IANS:

Üblicherweise, max Ausbelastung vorausgesetzt, zwischen 82-86\%. Sehr gut Ausdauertrainierte haben ihre IANS näher bei der Maxleistung, wenig Trainierte weiter davon entfernt. In absoluten Zahlen: IANS wird so korrigiert, dass sie im Laufen $2-3 \mathrm{~km} / \mathrm{h}$ und im Radsport 40-60 Watt unter der Maximalleistung liegt (entsprechend 82- 86\%).

\section{Borg:}

Üblicherweise, maximale Ausbelastung vorausgesetzt und geübt im Gebrauch der Borg-Skala, zwischen Borg 14-16.

Auch die rechnerischen Modelle haben sich entwickelt und eines davon, sinnigerweise modifizierte DmaxMethode genannt, hat Eingang ins neue Leistungsdiagnostik Manual Swiss Olympic gefunden. In der Grafik 2 und im nachfolgenden Text wird die Methode vorgestellt [36,4]. Sowohl die Literatur wie die bisherige praktische Erfahrung zeigen, dass es sich bei der modifizierten Dmax-Methode um eine objektive und reliable Bestimmungsmethode handelt, welche auch gute Korrelationen zu Wettkampfleistungen aufweist [37,36, 4].

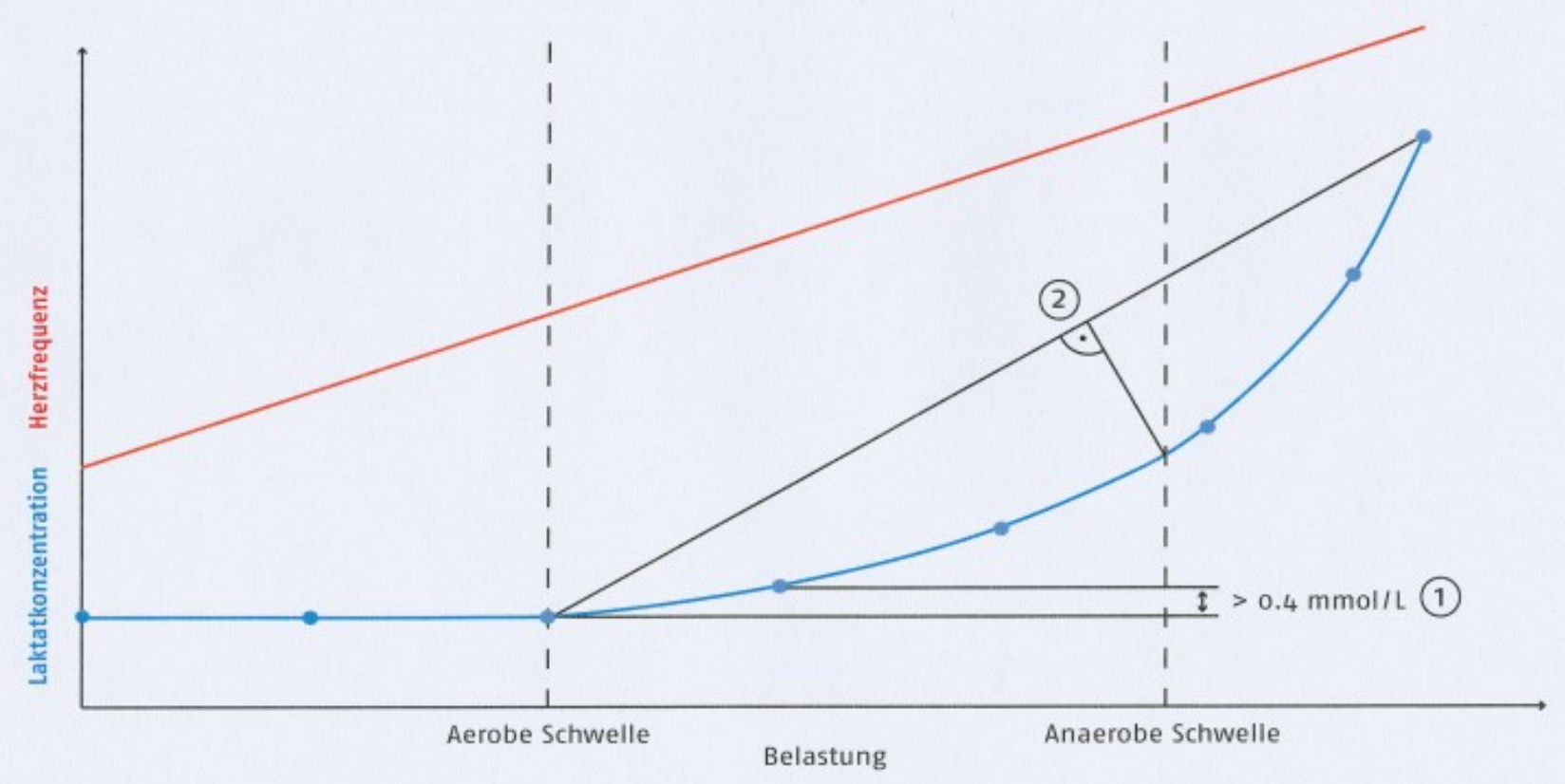

Grafik 3: Bestimmung der anaeroben Schwelle nach der modifizierten Dmax-Methode (Quelle: Leistungsdiagnostik Manual Swiss Olympic 2015; Abbildung 3-3. S. 28). 


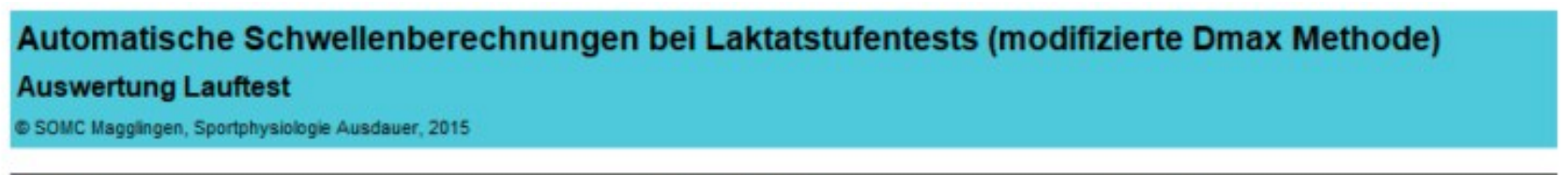

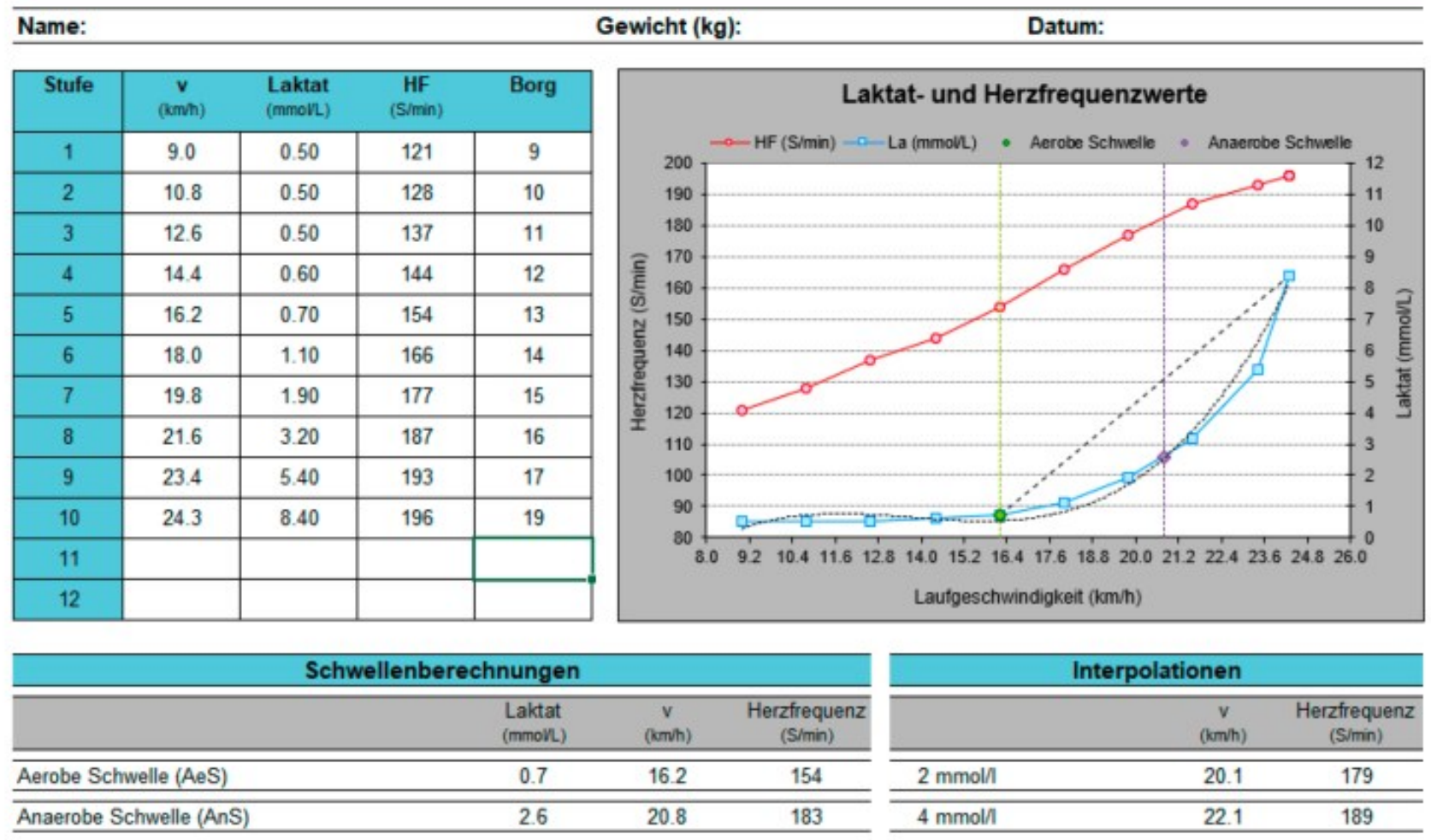

\section{Zur Ermittlung der anaeroben Schwelle wird folgendermassen vorgegangen (4):}

1. Bestimmung der aeroben Schwelle am Ende der Laktatbaseline. Die aerobe Schwelle entspricht derjenigen Geschwindigkeit oder Leistung, nach welcher der Anstieg der Laktatkonzentration zur folgenden Stufe erstmals $>0.4 \mathrm{mmol} / \mathrm{l}$ beträgt.

2. Der Punkt der anaeroben Schwelle auf der Laktatkurve wird mit einer Geraden mit dem Laktatwert bei Testabbruch verbunden.

3. Von dieser Geraden wird diejenige Senkrechte ermittelt, welche die maximale Distanz (=Dmax) zur Laktatkurve aufweist.

4. Die anaerobe Schwelle entspricht der Geschwindigkeit oder Leistung beim Schnittpunkt dieser Senkrechten auf der Laktatkurve.

Sowohl für die Bestimmungsmethode via IANS als auch die modifizierte Dmax-Methode braucht es idealerweise 6 bis 9 Stufen zur Ermittlung gleichvieler Messpunkte auf der Laktatkurve. Nur so kann die Baseline der Laktatkurve erkannt werden, also der Werte im Ruhelaktatbereich, welche trotz stufenweise erhöhter Belastung noch nicht ansteigen $[4,35]$.

Zur Ermittlung der anaeroben Schwelle mittels der modifizierten Dmax-Methode stehen auf der Swiss 
Olympic Website zwei frei zugängliche Excel-Files zur Verfügung, eines fürs Laufen und eines für den Radsport [38,39]. Diese sind in der Bedienung einfach und benutzerfreundlich. Hingegen bieten die einseitigen Dokumente keine direkte Hilfestellung für die Bestimmung der Trainingszonen und eignen sich auch nicht für die Trainingsberatung.

Welcher dieser beiden präsentierten Bestimmungsmethoden soll nun der Vorzug gegeben werden? Derweil der modifizierten Dmax-Methode eine mathematische Funktion zu Grunde liegt, ist sie bezüglich Reliabilität und eventueller späterer Publikation von Daten etwas im Vorteil. Sie bleibt dann allerdings starr, kann bei etwas undulierenden Laktatwerten, fehlender Laktat-Baseline oder auch Tagesschwankungen im Herzfrequenzbereich eine vermeintlich hohe, aber dann letztlich doch falsche Genauigkeit und Sicherheit suggerieren. Die IANS-Methode weist als Vorteile den Einbezug der Expertise des Testers und der Möglichkeit, Tagesschwankungen oder Variationen der physiologischen Parameter angemessen zu gewichten, auf. Hingegen braucht die Methode einiges an leistungsphysiologischer Erfahrung und würde für eine spätere Publikation von Daten gute Argumente brauchen. Unsere Erfahrung zeigt, dass beide Methoden meist ähnliche Werte liefern. Gerade bei kritischen Fällen scheinen beide Methoden, die eine in Ergänzung zur anderen, Zusatzinformationen für eine akkurate Bestimmung der anaeroben Schwelle zu liefern.

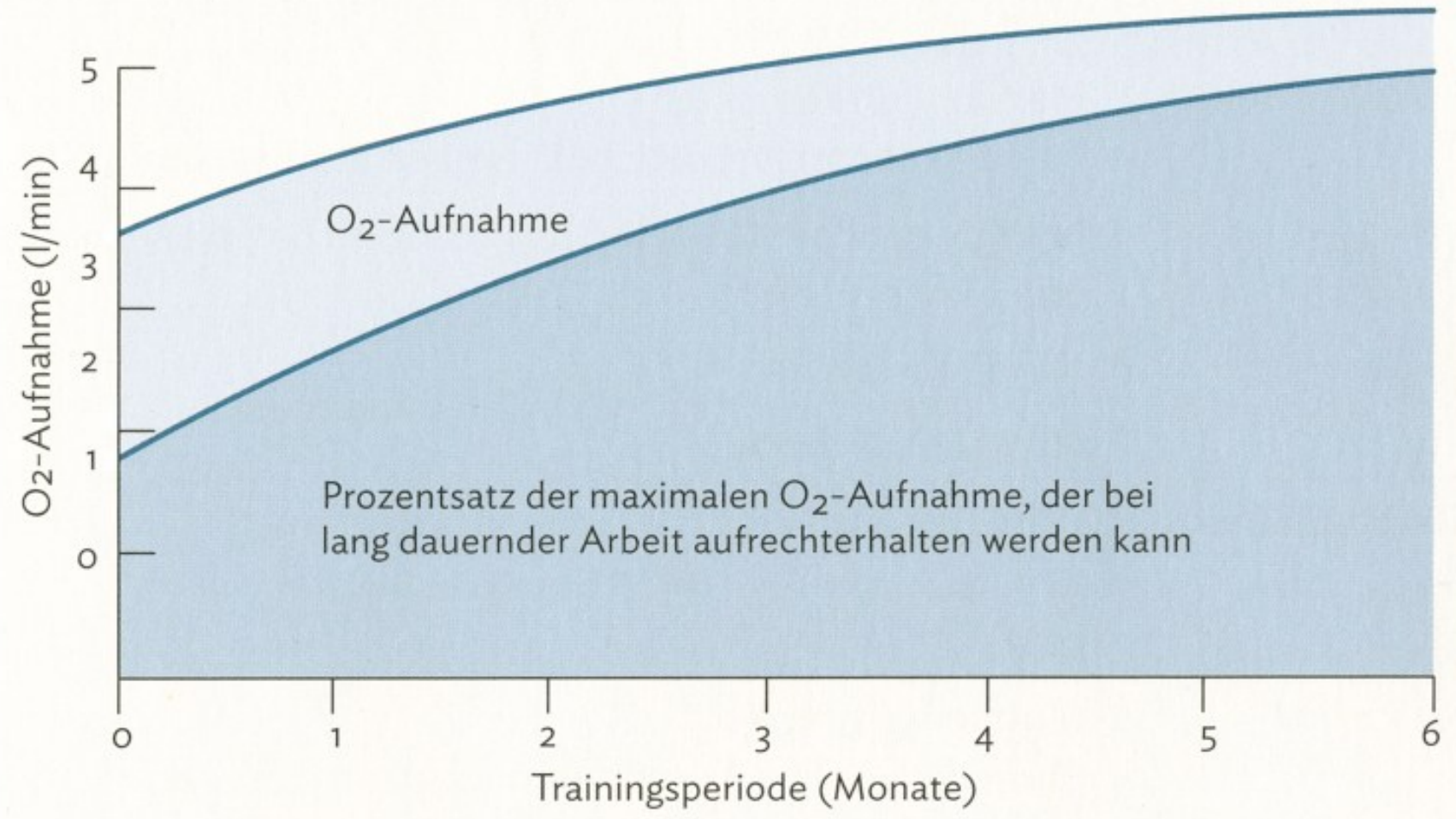




\section{$\mathrm{VO}_{2}$ max-Testung}

Welchen Stellenwert hat denn die $\mathrm{VO}_{2}$ max-Testung für den Ausdauersportler? Die sportartspezifisch ermittelte $\mathrm{VO}_{2}$ max gibt gut über das Potenzial des Ausdauersportlers Auskunft, möglicherweise auch im Rahmen einer gewissen Talentidentifikation. Sie lieferte bis vor einigen Jahren auch in der Perzeption der Athleten und leistungsphysiologisch versierten Trainern und Wissenschaftern keine Informationen zur Trainingssteuerung und hatte geringe Aussagekraft für den aktuellen Formstand. Dies hat diverse Gründe: Zum einen liefert das relativ kurze Protokoll mit dem zügigen Durchlaufen der Leistungsbereiche wirklich keinen Anhaltspunkt für die Trainingszonen, und zum andern ist die Messung aufgrund der relevanten biologischen und technischen Variation in der Messgenauigkeit anspruchsvoll. Die Grafik 5 illustriert sehr schön auch die Beziehung zwischen der $\mathrm{VO}_{2} \mathrm{max}$, also der maximalen Sauerstoffaufnahmefähigkeit, und der prozentualen Ausschöpfung derselben, also der anaeroben Schwelle, in Bezug auf die absolvierten Trainingsmonate [25). Es kann sehr gut erkannt werden, dass die anaerobe Schwelle sich entsprechend der steigenden Trainingsmonate schön verbessert, derweil die $\mathrm{VO}_{2}$ max sich auf einem Plateauwert einpendelt [25]. Dieses Phänomen entspricht sowohl der Literatur als auch den Beobachtungen von Athleten und Trainern. Die Leistungsfähigkeit bei der anaeroben Schwelle bzw. beim Testabbruch im Laktatstufentest korreliert für eine klassische Ausdauerdisziplin viel besser mit der aktuellen Leistungsfähigkeit als die $\mathrm{VO}_{2} \max [4,25,26,27,28]$.

Und trotzdem hat die $\mathrm{VO}_{2}$ max-Messung für den Leistungssport klar an Bedeutung gewonnen. Insbesondere die neuen Ausdauerformate mit den wenige Minuten dauernden Sprint-Formaten bilden die hochintensive Leistungsfähigkeit ab, wie sie in einer $\mathrm{VO}_{2}$ max-Testung gemessen wird. Des Weiteren kennen einige Sportarten, wie z.B. das olympische Cross-Country Mountain Biking, typische Wettkampfabläufe wie die hochintensive Startrunde von 4-6 min Dauer, in welcher die Fahrpositionen für den weiteren Wettkampf bezogen werden. Die Fähigkeit, für einige Minuten eine hochintensive Leistung erbringen zu können, ist wichtiger geworden. Nicht zuletzt muss an dieser Stelle erwähnt werden, dass die gezeigte Grafik 5 das Verhältnis zwischen $\mathrm{VO}_{2}$ max und anaerober Schwelle richtig abbildet. Hingegen kann die $\mathrm{VO}_{2}$ max eben sehr wohl trainiert und auch signifikant und je nach Trainingskonzept auch nachhaltig verbessert werden. Hier müssen vor allem erwähnt werden:

- HIIT, high intensity interval training, in zahlreichen Studien in praktisch allen Ausdauerdisziplinen konnte gezeigt werden, dass auch diese vormals magische Grenze der $\mathrm{VO}_{2}$ max nach oben verschoben werden kann [22,23,40,41].

- Mit sportartspezifischem Krafttraining kann aktive Arbeitsmuskulatur auf- und ausgebaut werden. So wird die maximale Kraftentwicklung, die Running bzw. Cycling economy, die spezifische maximale Leistungsfähigkeit und oft, aber nicht immer, auch die $\mathrm{VO}_{2} \max$ verbessert $[24,42,43,44]$.

- Höhentraining und dabei nachhaltige Formen mit Höhentrainingsketten können die $\mathrm{VO}_{2} \mathrm{max}$ durchaus und je nach Art der Intervention auch nachhaltig verbessern [14,15, 16,45].

Somit komplettiert die $\mathrm{VO}_{2}$ max-Messung für viele Ausdauer-Sportarten die Testbatterie. Gerade für die hochintensiven Trainings- und Wettkampfformen können Messwiederholungen der $\mathrm{VO}_{2}$ max zum intraund interindividuellen Längs- und Quervergleich wertvoll sein [40,41]. Bei aller Euphorie für die $\mathrm{VO}_{2} \max$ 
muss an dieser Stelle nochmals festgehalten werden: $\mathrm{VO}_{2} \max$ ist stark genetisch prädeterminiert, gewisse Verbesserungen sind möglich, der typische Messfehler ist relevant und sowohl die Abbruchleistung/anaerobe Schwelle eines Stufentests oder die später noch vorgestellte Leistung in einem Kapazitätstest sind aussagekräftigere Parameter als die $\mathrm{VO}_{2} \max [4,28,36]$.

Bezüglich des Protokolls ist es empfehlenswert, die Testdauer klar zu limitieren. Das Manual Leistungsdiagnostik Ausdauer spricht von 8-12 min [4]. Unsere Erfahrung zeigt, dass diese Zeit besser auf 5-8 min limitiert werden sollte, damit wirklich der maximale Plateauwert des $\mathrm{VO}_{2}$ unter noch steigender Belastung erreicht werden kann. Fürs Laufband ist zur Vermeidung einer motorischen Limitierung bei hohen Geschwindigkeiten und besseren Rekrutierung der laufspezifischen Muskelgruppen ein Steigungswinkel von z.B. 7\% sinnvoll, mit einem Belastungsinkrement von 0,5 km/h alle $30 \mathrm{sec}$. Für einen entsprechenden Radtest kann ein Inkrement von 25 Watt jede 30 sec oder 50 Watt jede 1 min sinnvoll sein. Die empfohlenen Anfangsbelastungen können dem Manual Swiss Olympic entnommen werden [4].

\section{Kapazitätstests}

Ein Kapazitätstest wird eingesetzt, wenn die aktuelle spezifische Leistungsfähigkeit, z.B. die reelle Wettkampfgeschwindigkeit einer Athletin erfasst werden soll. Keine indirekte andere Messmethode kann diese gleich valide abbilden. Als mögliche Formen hierzu wurden Testprotokolle entwickelt, welche entweder im Gelände oder im Labor mit dem eigenen Sportgerät im originalen Wettkampftempo absolviert werden. Zur Objektivierung werden als Erstes entweder der Zeitraum, wie lange diese Geschwindigkeit gehalten werden kann bzw. die Höhe der Geschwindigkeit ermittelt, welche der Athlet zu leisten vermag. Als Hilfsparameter werden im Gelände die Herzfrequenz, Borg und Laktat mitgemessen. Im Labor, z.B. auf dem grossen Laufband, können auch respiratorische Parameter $\left(\mathrm{VO}_{2}\right.$ und RER) helfen, um zu verstehen, ob diese Geschwindigkeit auch länger aufrechterhalten werden könnte. Der Kapazitätstest ist besonders aussagekräftig, weil er sich aus einer Kombination der isolierten physiologischen Faktoren zusammensetzt: Running bzw. Cycling economy, $\mathrm{VO}_{2} \mathrm{max}$, prozentuale Ausnutzung derselben, anaerobe Kapazität u.a.m. und dies in einer möglichst standardisierten Messung abbildet $[4,25]$.

Bei einem Kapazitätstest ist es sowohl im Gelände als auch im Labor möglich, sportspezifische Wettkampfbesonderheiten, wie z.B. eine rasche und höchstintensive Startrunde (wie z.B. beim olympischen MTB XC) nachzuahmen.

Die Wahl des Kapazitätstestes durch einen Sportverband kann auch Signalwirkung haben und das Trainingsverhalten der Athleten beeinflussen. Dies soll und kann bei der Neugestaltung des TestProtokolls, z.B. im Hinblick auf einen Zielwettkampf (EM, WM, Olympische Spiele) in speziellem Gelände oder spezifischer Belastungsdauer nachgeahmt werden [4].

\section{Wohin geht die Reise?}

Die in den vergangenen zwanzig Jahren akquirierten Erkenntnisse gilt es einzusetzen, die nun vorhandene Testbatterie zu brauchen und das Wissen an die aktuell aktive Generation von Athletinnen und Athleten, Trainerstab und Fachpersonen weiterzugeben und zu verbreiten. Des Weiteren sollen in den neu geschaffenen Sprintbereichen aktiv neue Testformen entwickelt werden, wie z.B. im 
Orientierungslaufen. Es bleibt diesbezüglich abzuwarten, ob diese doch veränderten Wettkampfformen zu einer weiteren Spezialisierung der Sportarten führen werden. Dabei wird es interessant sein zu beobachten, wie die nationalen Sportverbände sich aufstellen und die Athleten sich trainingsmässig vorbereiten und entscheiden. Hierbei sind die internationalen Sportverbände nicht nur gefordert, attraktive Formate fürs Publikum zu kreieren, sondern die Sportart als Ganzes, zusammen mit den nationalen Verbänden und ihren Athleten weiter zu entwickeln.

Der Mensch bleibt fasziniert durch Rekordmeldungen und Bestwerte. Das «Citius, Altius, Fortius»-Prinzip, welches sich der Sport selber gegeben hat, lässt grüssen - die 2-h-Marathon-Schallmauer ist nur ein Beispiel hierfür [46,47]. Betreffend dieses noch bevorstehenden, neuen Rekordes sind die Schlussfolgerungen der Autoren interessant und runden gleichermassen den vorliegenden Artikel ab: Dieser neue Marathonrekordhalter wird ein $\mathrm{VO}_{2} \max$ von $>85 \mathrm{ml} / \mathrm{kg}$ min haben und sollte davon $85 \%$ ausschöpfen können. Zusätzlich sollte er gezielt sportartspezifisches Krafttraining, Kapazitätsläufe, Schnelligkeitstraining und auch Einheiten oberhalb der anaeroben Schwelle absolvieren [47].

\section{Fazit für die Sportmedizinische Praxis / Zusammenfassung}

- Leistungsdiagnostische Tests bei Athleten und Patienten sind in der Hand des Sportmediziners/Sportwissenschafters ein wichtiges und wertvolles Tool zur Diagnostik und Trainingsberatung.

- Eine enge Zusammenarbeit zwischen Sportwissenschafter und Sportmediziner schafft für Athleten und Patienten wie die Fachpersonen eine Win-win-win-Situation.

- Je nach Anforderungsprofil der Sportart sind ein Laktatstufentest (Bestimmung der anaeroben Schwelle), eine Testung der $\mathrm{VO}_{2}$ max und der Leistung an der $\mathrm{VO}_{2}$ max sowie ein Kapazitätstest (Tempohärte mit Wettkampfbelastung) die Tests der Wahl.

- Alle Testungen sollen sportartspezifisch, valide und reliabel sein. Die Sportartspezifität hat für die praktische Aussagekraft der Testung am meisten Gewicht.

\section{Korrespondenzadresse}

\section{German Clénin}

Dr. med., Chief Medical Officer Swiss Ski Freestyle

Verbandsarzt Swiss Orienteering und

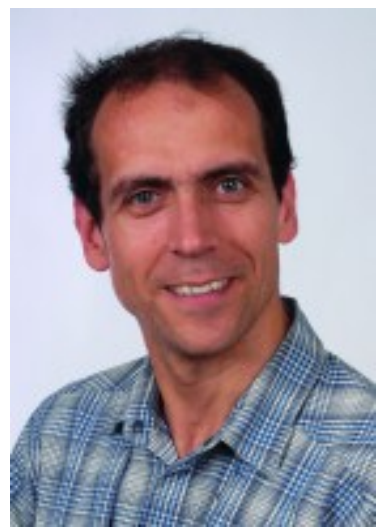


Swiss Athletics

Sportmedizinisches Zentrum Bern-Ittigen, Switzerland german.clenin@smzbich

\section{Referenzen}

1. Blair SN, Kampert JB, Kohl HW, III, Barlow CE, Macera CA, Paffenbarger RS, Jr, et al. Influences of cardiorespiratory fitness and other precursors on cardiovascular disease and all-cause mortality in men and women. JAMA (1996) 276:205-10.

2. Nystoriak MA, Bhatnagar A. Cardiovascular Effects and Benefits of Exercise. Front Cardiovasc Med. 2018 Sep 28;5:135.

3. Gleason PT, Kim JH. Exercise and Competitive Sport: Physiology, Adaptations, and Uncertain LongTerm Risks. Curr Treat Options Cardiovasc Med. 2017 Sep 14;19(10):79.

4. Leistungsdiagnostik Manual Swiss Olympic, 2015, Swiss Olympic, Ittigen, Schweiz, https://www.swissolympic.ch/dam/jcr:b15b191a-eb0d-46e8-b9c0-417b887a440d/Leistungsdiagnostik_ Manual_160201_DE.pdf.

5. Mujika Inigo. Endurance Training. Science and Practice. 2012, Publisher Inigo Mujika S.I.U. ISBN 978-84-939970-0-7. Vitoria-Gasteiz.Basque Country.

6. Nadelen M. Preparing for and playing in the heat. Jan 13, 2012. ACSM recommendations. Retrieved 28.2.2016.

7. Racinais $\mathrm{S}$ et al. Consensus recommendations on training and competing in the heat. Br J Sports Med, 2015 Sep;49(18):1164-73.

8. Schmit C, Le Meur Y, Duffield R, Robach P, Oussedik N, Coutts AJ, Hausswirth C. Heat-acclimatization and pre-cooling: a further boost for endurance performance? Scand J Med Sci Sports. 2017 Jan;27(1):55-65.

9. Clénin GE. Athletes'Health\&Top Performance. 2016, Publisher GE Clénin and Inhouse Publishing. ISBN 978-19-254972-4-3. Underwood, QLD, Australia.

10. Reilly T et al: Coping with Jet Lag : A position statement for the european college of sport science. European Journal of Sport Science, March 2007;7(1):1-7.

11. Svendsen IS, Taylor IM, Tønnessen E, Bahr R, Gleeson M. Training-related and competition-related risk factors for respiratory tract and gastrointestinal infections in elite cross-country skiers. $\mathrm{Br} \mathrm{J}$ Sports Med. 2016 Jul;50(13):809-15.

12. Wehrlin JP, Zuest P, Hallén J, Marti B. Live high-train low for 24 days increases hemoglobin mass and red cell volume in elite endurance athletes. J Appl Physiol (1985). 2006 Jun;100(6):1938-45.

13. Brugniaux JV, Schmitt L, Robach P, Nicolet G, Fouillot JP, Moutereau S, Lasne F, Pialoux V, Saas P, Chorvot MC, Cornolo J, Olsen NV \& Richalet JP (2006). Eighteen days of "living high, training low" stimulate erythropoiesis and enhance aerobic performance in elite middle-distance runners. J Appl Physiol 100, 203-211.

14. Brocherie F, Millet GP, Hauser A, Steiner T, Rysman J, Wehrlin JP \& Girard O (2015b). "live high-train low and high” hypoxic training improves team-sport performance. Med Sci Sports Exerc 47, 2140-2149.

15. Saugy JJ, Schmitt L, Hauser A, Constantin G, Cejuela R, Faiss R, Wehrlin JP, Rosset J, Robinson N \& Millet GP (2016). Same performance changes after live high-train low in normobaric vs. hypobaric hypoxia. Frontiers in physiology 7, 138.

16. Wehrlin JP, Marti B, Hallén J. Hemoglobin Mass and Aerobic Performance at Moderate Altitude in Elite Athletes. Adv Exp Med Biol. 2016;903:357-74.

17. Seiler S. What is best practice for training intensity and duration distribution in endurance athletes. Int J Sports Physiol Perform. 2010 Sep;5(3):276-91. 
18. Muñoz I, Seiler S, Bautista J, España J, Larumbe E, Esteve-Lanao J. Does polarized training improve performance in recreational runners? Int J Sports Physiol Perform. 2014 Mar;9(2):265-72

19. Tønnessen E, Sylta $\emptyset$, Haugen TA, Hem E, Svendsen IS, Seiler S. The road to gold: training and peaking characteristics in the year prior to a gold medal endurance performance. PLoS One. 2014 Jul 14; 9(7): e101796.

20. Stöggl TL, Sperlich B. The training intensity distribution among well-trained and elite endurance athletes. Front Physiol. 2015 Oct 27;6:295.

21. Coe, Sebastian (2013). Running My Life. Hodder. pp. 38, 39. ISBN 978-1-444-73253-5.

22. Tabata, Izumi; Nishimura, Kouji; Kouzaki, Motoki; Hirai, Yuusuke; Ogita, Futoshi; Miyachi, Motohiko; Yamamoto, Kaoru (1996). "Effects of moderate-intensity endurance and high-intensity intermittent training on anaerobic capacity and $\mathrm{VO}_{2}$ max". Medicine \& Science in Sports \& Exercise. 28 (10): 1327-30.

23. Milanovic' Z, Sporiš G, Weston M. Effectiveness of High-Intensity Interval Training (HIT) and Continuous Endurance Training for $\mathrm{VO}_{2}$ max Improvements: A Systematic Review and Meta-Analysis of Controlled Trials. Sports Med. 2015 Oct;45(10):1469-81.

24. Beattie K, Carson BP, Lyons M, Rossiter A, Kenny IC. The Effect of Strength Training on Performance Indicators in Distance Runners.

J Strength Cond Res. 2017 Jan;31(1):9-23.

25. Hegner J. Training fundiert erklärt. Handbuch der Trainingslehre. März 2015, 6. Auflage, Ingold Verlag, Schweiz.ISBN 978-3-03700-207-0.

26. Hopkins WG, Schabort, EJ \& Hawley JA. Reliability of power in physical performance tests. Sports Med. 2001. 31.211-234.

27. Bentley DJ, McNaughton LR, Thompson D, Vleck VE \& Batterham AM. Peak power output, the lactate threshold, and time trial performance in cyclists. Med Sci Sports Exerc 2001. 33,2077-2081.

28. Clénin GE. Prädiktiver Wert von vier unterschiedlich aufwendigen Ausdauertests für die 15kmWettkampfleistung von Frauen und Männern mit ähnlichem Dauerleistungsvermögen. Inauguraldissertation der Med. Fakultät der Uni ZH. Zürich 1997.

29. Hill DW. The critical power concept. A review. Sports Med. 1993 Oct;16(4):237-54.

30. Allen H \& Coggan, A. Training and racing with a power meter. VeloPress, Boulder, Colorado, USA, 2010.

31. Poole DC, Burnley M, Vanhatalo A, Rossiter HB, Jones AM. Critical Power : An Important Fatigue Threshold in Exercise Physiology. Med Sci Sports Exerc.2016 Nov;48(11):2320-2334.

32. Jones AM, Vanhatalo A. The 'Critical Power' Concept : Applications to Sports Performance with a Focus on Intermittent High-Intensity Exercise. Sports Med. 2017 Mar;47(Suppl 1):65-78.

33. Jones, A.M. \& Doust, J.H. A 1\% treadmill grade most accurately reflects the energetic cost of outdoor running. J Sports Sci 14,321-327 (1996).

34. Mader, A., et al. Zur Beurteilung der sportartspezifischen Ausdauerleistungsfähigkeit im Labor. Sportarzt Sportmed 27, 80-88,109-112 (1976).

35. Tschopp M. Manual Leistungsdiagnostik Ausdauer, SOMC Davos, Leukerbad, Magglingen, Muttenz, Zürich. Qualitätsentwicklung Sportmed Swiss Olympic, Magglingen. Februar 2003.

36. Bishop D, Jenkins DG, Mackinnon LT. The relationship between plasma lactate parameters, Wpeak and 1-h cycling performance in women. Med Sci Sports Exerc. 1998 Aug;30(8):1270-5.

37. Fabre N, Balestreri F, Pellegrini B, Schena F. The modified Dmax method is reliable to predict the second ventilatory threshold in elite cross-country skiers. J Strength Cond Res. 2010 Jun;24(6):1546-52.

38. Excel-File zur Auswertung modDmax-Laufen, download am 23.2.2019:

https://www.swissolympic.ch/dam/jcr:48fd8f9d-e790-41fd-b0c0-6f078af9d038/Laktatstufentests_modD max_Schwellen

berechnungen_Lauf_DE.xlsxxcel-File. 
39. Excel-File zur Auswertung modDmax-Radsport, download am 23.2.2019:

https://www.swissolympic.ch/dam/jcr:413912f7-0e02-4e3a-afd5-f10371b11d07/Laktatstufentests_mod Dmax_Schwellen berechnungen_Rad_DE.xlsx

40. Helgerud J, Høydal K, Wang E, Karlsen T, Berg P, Bjerkaas M, Simonsen T, Helgesen C, Hjorth N, Bach $\mathrm{R}$, Hoff J. Aerobic high-intensity intervals improve $\mathrm{VO}_{2}$ max more than moderate training. Med Sci Sports Exerc. 2007 Apr;39(4):665-71.

41. Billat LV, Koralsztein JP. Significance of the velocity at $\mathrm{VO}_{2} \max$ and time to exhaustion at this velocity. Sports Med, 1996 Aug:22(2):90-108.

42. Rønnestad BR, Hansen EA, Raastad T. In-season strength maintenance training increases well-trained cyclists’ performance. Eur J Appl Physiol. 2010 Dec;110(6):1269-82.

43. Rønnestad BR, Mujika I. Optimizing strength training for running and cycling endurance performance: A review. Scand J Med Sci Sports. 2014 Aug;24(4):603-12.

44. Rønnestad BR, Hansen J, Nygaard H. 10 weeks of heavy strength training improves performancerelated measurements in elite cyclists. J Sports Sci. 2017 Jul;35(14):1435-1441.

45. Millet GP, Roels B, Schmitt L, Woorons X, Richalet JP. Combining hypoxic methods for peak performance. Sports Med. 2010 Jan 1;40(1):1-25.

46. Haugen T, Paulsen G, Seiler S, Sandbakk O. New Records in Human Power. Int J Sports Physiol Perform, 2018 Jul 1:13(6):678-686.

47. Sousa CV, Sales MM, Nikolaidis PT, Rosemann T, Knechtle B. How much further for the sub-2-hour marathon? Open Access J Sports Med 2018 Jul 31;9:139-145.

\section{AEROBIC CAPACITY TESTING ANAEROBIC THRESHOLD BIATHLON CROSS COUNTRY \\ SKIING CYCLING ENDURANCE EXERCISE TESTING INCREMENTAL LACTATE \\ TESTING ORIENTEERING ROWING RUNNING SWIMMING TRAINING ZONES TRIATHLON VOロMAX}

